\section{Qualitative synthesis}

\section{Parents and informal caregivers feel they receive insufficient communication about routine childhood vaccination}

10.1136/eb-2017-102685

\section{Victoria Niederhauser}

University of Tennessee Knoxville College of Nursing, Knoxville, Tennessee, USA

Correspondence to: Professor Victoria Niederhauser, University of Tennessee Knoxville, College of Nursing, 1200 Volunteer Blvd, Knoxville, TN, 37996; vniederh@utk.edu

Commentary on: Ames HM, Glenton C, Lewin S, et al. Parents' and informal caregivers' views and experiences of communication about routine childhood vaccination: a synthesis of qualitative evidence. Cochrane Database Syst Rev 2017;2:CD011787.

\section{Implications for practice and research}

- This study sheds light on the importance of providers, public health organisations and others to provide in-depth, detailed, frequent and timely vaccination communication via a variety of different sources to parents and caregivers of young children.

- Only 7 of the 43 findings were related to the association between vaccination communication and decisions, and most of these indicated a low to moderate CI. There is a need to further research vaccination communications as an intervention by a variety of sources (individual provider, public health, media and so on) and the impact on parents' and caregivers' vaccination decisions.

- Further research is needed to examine the relationship of communication and vaccination decisions in lower resource countries.

\section{Context}

Providing parents or primary caretakers with information about childhood vaccinations has been a long-standing practice for healthcare providers, public health, vaccine manufacturers and others. There are multiple methods of vaccination communication, including public health campaigns, individual counselling, advertising and the mass media, and the type of information provided about vaccinations varies among these sources. Many individual studies have sought to understand the relationship between vaccination communication and the decision of parents and caretakers to vaccinate their children. This study by Ames and colleagues seeks to understand parents' and caregivers' experiences with communication regarding childhood vaccinations and if there is a relationship between communication and decisions to vaccinate their children.

\section{Methods}

This systematic review included 38 qualitative primary studies that focused on the vaccination communication experiences and decisions of parents and caregivers of children aged 6 years and younger. After an extensive literature search using multiple online databases, the research team identified 79 full-text articles that met the inclusion criteria, and then used purposeful sampling to pare the number of article to include in the final study to 38. Qualitative findings were synthesised using thematic analysis and constant comparison described by Miles et al. ${ }^{1}$ Furthermore, the synthesis included examining related Cochrane Reviews finding using a matrix model.

\section{Findings}

Six major themes with varying confidence levels emerged from the analysis, namely timing of vaccine information, availability of vaccination information, amount of vaccination information, source of vaccination information, content of vaccination information, and the relationship between vaccination information and vaccinations decisions. Among these six themes, there was strong evidence that the parents and caregivers felt they received an insufficient amount of vaccination information. In addition, they did not feel the vaccination information was provided in a balanced manner and they were unsure of how to determine reputable, trusting sources of vaccination information. Parents and caregivers felt healthcare providers were an important sources of vaccination information and they expected to spend quality time with their provider (in a non-hurried manner, with the ability to ask questions freely, with a provider who is open and receptive to concerns) openly discussing specifics about childhood vaccinations.

\section{Commentary}

Worldwide, vaccination rates in children are suboptimal. ${ }^{2}$ Through this synthesis of published qualitative studies, we increase our understanding of the types, amount, timing, sources and methods of vaccination communication that parents and caregivers desire and expect from providers, public health organisations and others. This review provides a summary of what is known and the gaps in knowledge about the relationship between communication and vaccination decisions. 


\section{Child health}

In clinical practice, healthcare providers and public health officials communicate on a routine basis about the positive impact that vaccinations have on health, including mass media campaigns and regularly providing information to parents and caregivers prior to immunising children. Despite all of these communications, we are still experiencing major outbreaks of vaccine-preventable diseases in children throughout the world. Given the evidence presented in this study, there are several significant findings that indicate the current practice of providing vaccination information may not be enough. These findings validate the need to provide parents and caretakers with more childhood vaccination information in an unhurried, unbiased and balanced (risks and benefits) manner. Also, exploring creative and different ways and venues to provide vaccination information to parents and caretakers must be tried and tested.

The results of this systematic review allow us to glean important information about the relationship between vaccination communication and vaccination decisions. We understand more about what parents and caregiver want in the way of communication. However, the important relationship between having the desired amount, type, source of communication and the decision to vaccinate remains vague, at best. The researchers acknowledge that a majority of the studies were in higher resources countries and that they omitted studies that were written not in languages of the researchers (English, French and Scandinavian), and therefore generalisability of these findings should be interpreted with caution.

Competing interests None declared.

Provenance and peer review Commissioned; internally peer reviewed.

(c) Article author(s) (or their employer(s) unless otherwise stated in the text of the article) 2017. All rights reserved. No commercial use is permitted unless otherwise expressly granted.

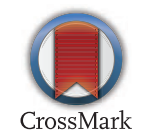

\section{References}

1. Miles MB, Huberman AM, Saldana J. Qualitative data analysis. A method sourcebook. 3rd rev edn. California: Sage, 2014.

2. World Health Organization (WHO). Immunization Coverage. http://www.who.int/ mediacentre/factsheets/fs378/en/ (accessed 28 Apr 2017). 